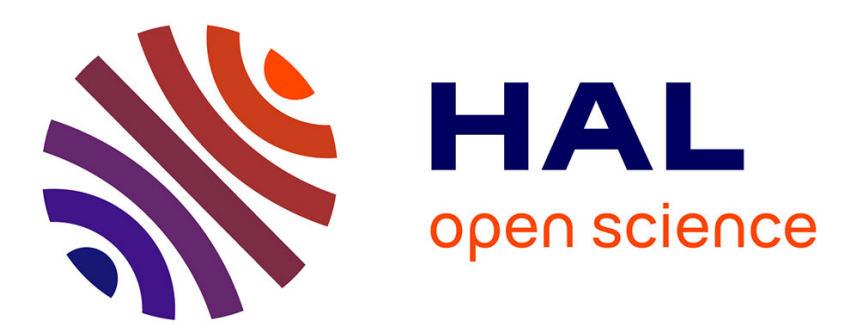

\title{
Open-Loop Tests and Validation of a New Two-Wheeled Vehicle Riding Simulator
}

Lamri Nehaoua, Salim Hima, Hichem Arioui, Nicolas Seguy, Stéphane Espié

\section{To cite this version:}

Lamri Nehaoua, Salim Hima, Hichem Arioui, Nicolas Seguy, Stéphane Espié. Open-Loop Tests and Validation of a New Two-Wheeled Vehicle Riding Simulator. European Control Conference (ECC'07), Jul 2007, Kos, Greece. pp.4911-4916. hal-00342978

\section{HAL Id: hal-00342978 https://hal.science/hal-00342978}

Submitted on 1 Jul 2009

HAL is a multi-disciplinary open access archive for the deposit and dissemination of scientific research documents, whether they are published or not. The documents may come from teaching and research institutions in France or abroad, or from public or private research centers.
L'archive ouverte pluridisciplinaire HAL, est destinée au dépôt et à la diffusion de documents scientifiques de niveau recherche, publiés ou non, émanant des établissements d'enseignement et de recherche français ou étrangers, des laboratoires publics ou privés. 


\title{
Open-Loop Test and Validation of a New Two-Wheeled Vehicle Riding Simulator
}

\author{
L. Nehaoua, S. Hima, H. Arioui, N. Séguy and S. Éspié
}

\begin{abstract}
This paper highlights the mechanical and mechatronic properties of a two-wheeled driving simulator. It gives also the important motivation which facilitates the specification movement and inertial effect choice.

Considering the principles purpose of this simulator:

- as a training tool for new riders with different scenarios: normal traffic environment, dangerous riding situations (avoidance, emergency braking, nearly failling or slipping situations, bad weather conditions, etc.)

- to study riders behaviours in such situations

the study has lead to an original 5 degree of freedom (DOF). These mobilities consisting in roll, yaw, pitch and 2DOF applied on the handlebar. The kinematics of the platform is lightly described. Finally, some performances results are shown validating the initial requirement.
\end{abstract}

\section{INTRODUCTION}

The road safety of the two motorized wheels vehicle has been neglected for a long time. Whereas the number of car driver killed in road accidents dropped, the number of killed motorcyclists unfortunately increased [1]. For this reason, the research institutions endeavour as soon as possible to bring adapted answers to the problem of motorcyclists safety by developing works on motorcycle riders, whose risk of fatal accident is much higher compared to car drivers.

Specificities and difficulties of the two wheels vehicle driving are, initially, of a dynamic nature: equilibrium, control, manoeuvrability, braking and reaction to emergencies. Finally, the problem of visibility is particularly critical especially in situations of intersection or overtaking. About behaviors, it has to be notice that riders underestimate the motorcycle dynamics and often ride at speeds which does not permits to face the unforeseen situations.

Driving simulators are efficient tools destinated to realize the different studies in a safe environment. Its purpose is to reproduce riding sensations in a constrained environment and makes possible to train novices as well as experienced riders. In the past, some motorcycle driving simulators were build. The first prototype was developed by Honda in 1988 , with the purpose of studying the motorcycle stability and manoeuvrability [2]. It consists of a platform with five degrees of freedom (lateral, roll, yaw, pitch and handlebar steering) actuated by seven actuators. After 1990, Honda launches out a second prototype of simple simulators with three DOF (roll, pitch and steering), to allow the safe trainee to experience

L.Nehaoua and S. Éspié: INRETS, 2 Av Gl Malleret-Joinville, 94114, Arceuil, France. email: nehaoua, espie@inrets.fr

N. Séguy and H. Arioui: IIBISC LSC-LAMI CNRS FRE 2873, 40 rue Pelvoux 91020, Evry, France. email: seguy, arioui, himaliup.univ-evry.fr hazardous situations after various traffic conditions on the real city roads [3]. A third prototype was developped using a six DOF parallel manipulator to plan the motion of the platform, and a head mounted display (HMD) for visual projection [4].

The MORIS simulator project was started by the PERCRO Laboratory in 1995, with the aim of developing a tool for designers to acquire knowledge on motorcycle handling and stability [5]. It consists of a real scooter mock-up mounted on a Stewart parallel platform with seven DOF (include steering axis).

Finally, at the University of Padova, a motorcycle simulator prototype has been designed, in order to study the manvehicle interaction and develop a rider control model [6]. The mechanical platform is a serie structure with five DOF (lateral, roll, pitch, yaw and steering) actuated electrically.

\section{Preliminaries}

In this project, we aim to conceive a mechanical platform for the training and the behavioral study of the two wheels vehicle users. We look to reproduce the important inertial effects perceived for the application needs but not all the motorcycle movements. So, the real amplitude of the various DOF was not a dominating object during the design phase. Then, the number of DOF privileged for our architecture is determined by the simulator's application. After several investigations, three rotations were privileged:

- roll: for the reproduction of short cornering (slalom, way changes),

- pitch: to restitute the accelerations movements illusion as well as fork movements,

- yaw: to reproduce the rear motorcycle wheel skid like in close accident situations. The skid of the front wheel is not reproduced because it is immediately fatal.

Otherwise, the multiplication of the perception stimuli strongly increases the riding simulation sensations [7]. Based on this idea, a double haptic feedback is implemented on the handlebar. The first one enables to restitute an inertial delay on the rider bust during the acceleration and braking phases. An effort is created on the motorcyclist arms while varying the distance between the saddle and the handlebar. The second force feedback have the aim to restitute the torque resulting from the tire - road contact, gyroscopic effects and of the front wheel trail [8].

The position of the various rotation axes is of great importance. No psychophysical study was carried out in the literature except some very simple cases [9]. Therefore, these axes are taken starting from the real motorcycle kinematics 
[10]. In order to produce the necessary yaw to feel the rear wheel skid, a slide is positioned on the back of the motorcycle frame. The roll axis is taken in the motorcycle symmetry plane with an ajustable height in order to test several configurations and to find the best perception results.

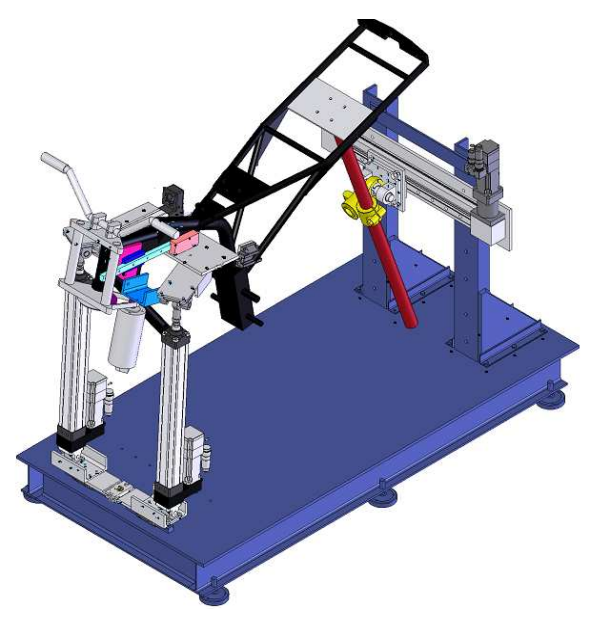

Fig. 1. CAD model of the simulator platform

Lastly, for the pitch axis, it is the deplacement of the front fork in the acceleration and braking phases which was privileged, therefore the axis passes by the back of the motorcycle frame. Figure 1 represents the CAD model of the platform simulator which will be described in the next sections.

\section{PLATFORM KINEMATICS}

From the various considerations cited in the previous section, the mechanical layout of the simulator's platform was established (figure 2). The proposed architecture has:

- 3 spherical joints $\left(P_{1}, P_{2}\right.$ and $\left.O_{3}\right)$ with 3 rotations each,

- 1 prismatic joint $O_{3}$ with one translation DOF for the yaw motion,

- 2 legs $\left(\overrightarrow{O_{1} P_{1}}\right.$ and $\left.\overrightarrow{O_{2} P_{2}}\right)$ with one prismatic joint each for roll and pitch motion,

- 2 cylindrical joints $\left(O_{1}\right.$ and $\left.O_{2}\right)$ with 1 rotation and 1 translation each,

- 1 constraint to insure symmetric displacements of points $O_{1}$ and $O_{2}$ about $(x O z)$ plane.

Using the general formula of Grubler which gives the mobility $m$ of a spacial mechanism by:

$$
m=I_{c}-6(l-n+1)
$$

where, $I_{c}$ is the number of kinematics unknowns, $l$ the number of links, $n$ the number of platform solides. Hence, we have 16 kinematics unknowns, 8 links and 7 bodies, so $m=4$. The constraint leeds to a final mobility of 3 . This mobilities are all actuated which allow to realize the three rotation movements (roll, pitch and yaw).

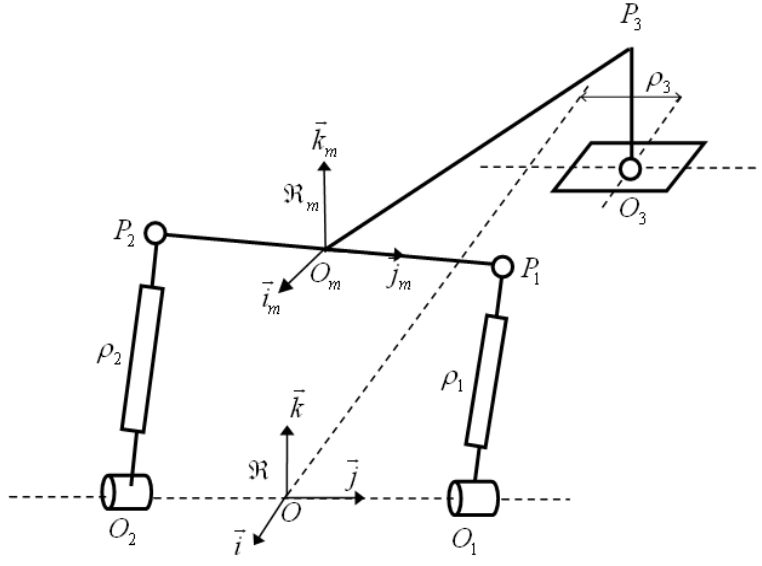

Fig. 2. Kinematics sketch of the simulator platform

The actual configuration of the motorcycle chassis frame is given by the position and the orientation of the mobile reference $\Re_{m}\left(O_{m}, \vec{i}_{m}, \vec{j}_{m}, \vec{k}_{m}\right)$ with respect to the inertial reference $(O, \vec{i}, \vec{j}, \vec{k})$. The inverse kinematics makes possible to transform these position and orientation in articular coordinates in order to drive the various actuators. Because only three among the six position and orientation parameters of the mobile platform of this mechanism are independent, the origin point $O_{m}$ of the mobile reference can be located either by the orientation angles $(\varphi, \theta, \psi)$ which are respectively roll, pitch and yaw rotations. Moreover, for the motion cueing algorithms consideration (as will be explained in section $I V$ $D)$, we adopt the rotation angles representation. To do this, we must express the mobile reference frame position vector $O O_{m}$ as a function of orientation angles. For this, we define four intermediate frame references; $\Re_{1}\left(\vec{i}_{1}, \vec{j}_{1}, \vec{k}_{1}\right)$ obtained by translating the frame $\Re$ from $O$ to $O_{3}, \Re_{2}\left(\vec{i}_{2}, \vec{j}_{2}, \vec{k}_{2}\right)$ by tilting the frame $\Re_{1}$ about the $\vec{j}_{1}$ axis, $\Re_{3}\left(\vec{i}_{2}, \vec{j}_{2}, \vec{k}_{2}\right)$ by yawing the frame $\Re_{2}$ about $\vec{k}_{2}$ axis and $\Re_{4}\left(\vec{i}_{4}, \vec{j}_{4}, \vec{k}_{4}\right)$ by rolling the frame $\Re_{3}$ about $\vec{i}_{3}$ axis as shown in figure 3 .

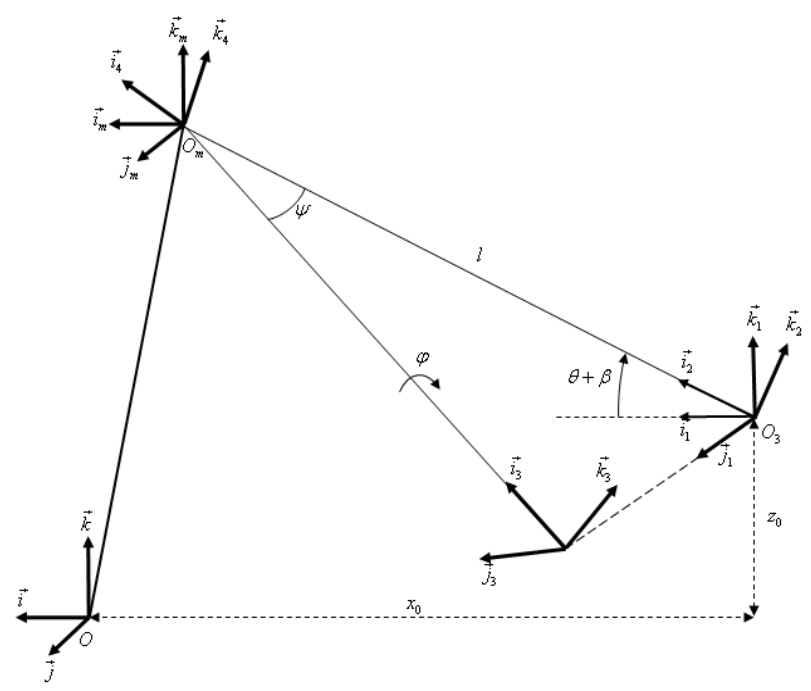

Fig. 3. Reference frame configuration 
We write than the following transformations for $i=1,2$ :

$$
\begin{aligned}
& O_{i} P_{i}=O_{i} O+O P_{i} \\
& O P_{i}=O O_{3}+O_{3} O_{m}+O_{m} P_{i}
\end{aligned}
$$

where:

$O_{i} O=\left(\begin{array}{c}0 \\ \mp d \\ 0\end{array}\right), O O_{3}=\left(\begin{array}{c}x_{0} \\ \rho_{3} \\ z_{0}\end{array}\right)$

and

$$
\begin{aligned}
& O_{3} O_{m}=R . O_{3} O_{m}^{R_{4}} \\
& O_{m} P_{i}=R . O_{m} P_{i}^{R_{4}}
\end{aligned}
$$

$d$ is the variable distance between points $O$ and respectively $O_{1}$ and $O_{2}, \rho_{3}$ is the lateral displacment of the point $O_{3}$ and $R$ is the rotation matrix which expresses the orientation of the mobile reference $\Re_{4}$ with respect to the fixed reference $\Re$ :

$$
R=R_{\theta} R_{\psi} R_{\varphi}
$$

$\theta, \psi$ and $\varphi$ are respectively the tilt, yaw and roll angles. After developping, we find the expression of vectors $O O_{m}$ and $O P_{i}$ :

$$
O O_{m}^{R}=\left(\begin{array}{c}
x_{0}+l c(\theta+\beta) c \psi \\
l s \psi+\rho_{3} \\
z_{0}-l s(\theta+\beta) c \psi
\end{array}\right)
$$

and

$$
O_{m} P_{i}^{R}=\left[\begin{array}{c} 
\pm l_{m}(-c(\theta+\beta) s \psi c \varphi+s(\theta+\beta) s \varphi) \\
\pm l_{m} c \psi c \varphi \\
\pm l_{m}(s(\theta+\beta) s \psi c \varphi+c(\theta+\beta) s \varphi)
\end{array}\right]
$$

The mechanical constraint impose that in the fixed reference $\Re$, the $y$ component of the vector $O O_{m}$ is equal to zero and the two points $P_{i}$ and $O_{i}$ have the same $y$ components. From this, we can deduce the displacements $\rho_{3}$ and $d$ :

$$
\begin{aligned}
& \rho_{3}=-l \sin \psi \\
& d=l_{m} \cos \psi \sin \varphi
\end{aligned}
$$

Now, replacing equations (5) and (6) into equation (2), we can deduce the position vector $O_{i} P_{i}$ expressed in the inertial reference $\Re$.

From the expression of the two vectors $O_{i} P_{i}$ of equation (2), we can calculate the two legs stroke $\rho_{i}$ with $i=1,2$. Therefore, we can write:

$$
\rho_{i}^{2}=O_{i} P_{i}^{T} \cdot O_{i} P_{i}
$$

Equations (7) and (8) constitute the so called Inverse Geometric model, which gives the articular coordinates $\rho_{1}$, $\rho_{2}$ and $\rho_{3}$ as a function of the mobile platform orientation $(\theta, \psi, \varphi)$.

After establishing the inverse geometry model, we look to determine the legs actuators velocity $\rho_{i}$ from the upper platform angular velocity $\omega$, which means to express the so called Inverse Kinematics of the platform, then we have for $i=1,2$ :

$$
\dot{\rho}_{i}=\dot{O_{i} P_{i}} \bullet n_{i}
$$

where $n_{i}=\frac{O_{i} P_{i}}{\rho_{i}}$ is the unit vector along the two legs and the operator $\bullet$ is the scalar product. By derivating equation (2) we find:

$$
\begin{aligned}
& \dot{O_{i}} P_{i}=\dot{O} \dot{O}+\dot{O P} \dot{P}_{i} \\
& \dot{O P} \dot{P}_{i}=\dot{O O} \dot{O}_{3}+\dot{O}_{3} \dot{O}_{m}+O_{m} P_{i}
\end{aligned}
$$

where:

$$
\dot{O_{i} O}=\left(\begin{array}{c}
0 \\
\mp \dot{d} \\
0
\end{array}\right), \dot{O O_{3}}=\left(\begin{array}{c}
0 \\
\dot{\rho}_{3} \\
0
\end{array}\right)
$$

and

$$
\begin{aligned}
& O_{3} \dot{O}_{m}=\omega^{R} \times R . O_{3} O_{m}^{R_{4}} \\
& O_{m} P_{i}=\omega^{R} \times R . O_{m} P_{i}^{R}{ }^{R} \\
& \omega=\dot{\theta} \vec{j}_{1}+\dot{\psi} \vec{k}_{2}+\dot{\varphi} \vec{i}_{3}
\end{aligned}
$$

By transforming the vectors $\vec{k}_{2}$ and $\vec{i}_{3}$ in the fixed frame $\Re(\vec{i}, \vec{j}, \vec{k})$, we write the expression of $\omega^{R}$ as following:

$$
\omega^{R}=\left(\begin{array}{ccc}
0 & s(\theta+\beta) & c(\theta+\beta) c \psi \\
1 & 0 & s \psi \\
0 & c(\theta+\beta) & -s(\theta+\beta) c \psi
\end{array}\right)\left(\begin{array}{c}
\dot{\theta} \\
\dot{\psi} \\
\dot{\varphi}
\end{array}\right)
$$

After developping, we can formulate the inverse kinematics in the form:

$$
\left(\begin{array}{c}
\dot{\rho}_{1} \\
\dot{\rho}_{2} \\
\dot{\rho}_{3}
\end{array}\right)=J\left(\begin{array}{c}
\dot{\theta} \\
\dot{\psi} \\
\dot{\varphi}
\end{array}\right)
$$

where $J$ is a $9 \times 9$ Jacobian matrix, $\dot{\rho}_{1}, \dot{\rho}_{2}$ are determined from equation (9) and $\dot{\rho}_{3}=-l \dot{\psi} \cos \psi$

\section{Simulator SOFTwARE ARCHITECTURE}

Based on the $\mathrm{SIM}^{2}$ car driving simulator software architecture [11] [12], some adaptation are carried out to close the simulation loop of the present riding motorcycle simulator (figure 4). This software platform is organized around an interfacing system and two PCs linked with TCP/UDP communication protocole. The first PC is dedicated to the motorcycle dynamic model and the traffic management (ARCHISIM PC) and the second one for the motion planning and cueing control.

\section{A. Acquisition's electronic}

An interface board is used for the low management level of the simulators mock-up. It is based on an industrial microcontroller. It aims to acquire data from the driver commands to send the resulting signals to the ARCHISIM PC via the CAN network protocole communication. After updating the motorcycle states, informations on the engine mode and motorcycle speed are sent from the traffic managment module towards the acquisition system to be displayed on the mock-up dashboard. 


\section{B. Archisim PC}

1) Motorcycle dynamic model: It deals with the calcul of the motorcycle dynamics based on the different driver actions (throttle, brake and clutch levers, gearbox selector) transmitted by the acquisiton system. For our applications (training and risk sensitization) a simple Sharp motorcycle dynamic model [13] is used with some adaptations. For the longitudinal dynamics, a one DOF model is developed which determines the longitudinal acceleration and traveling velocity of the virtual motorcycle from the throttle, clutch lever and the transmission state. The resulting velocity and the exerted rider torque on the handlebar serve as input paramete

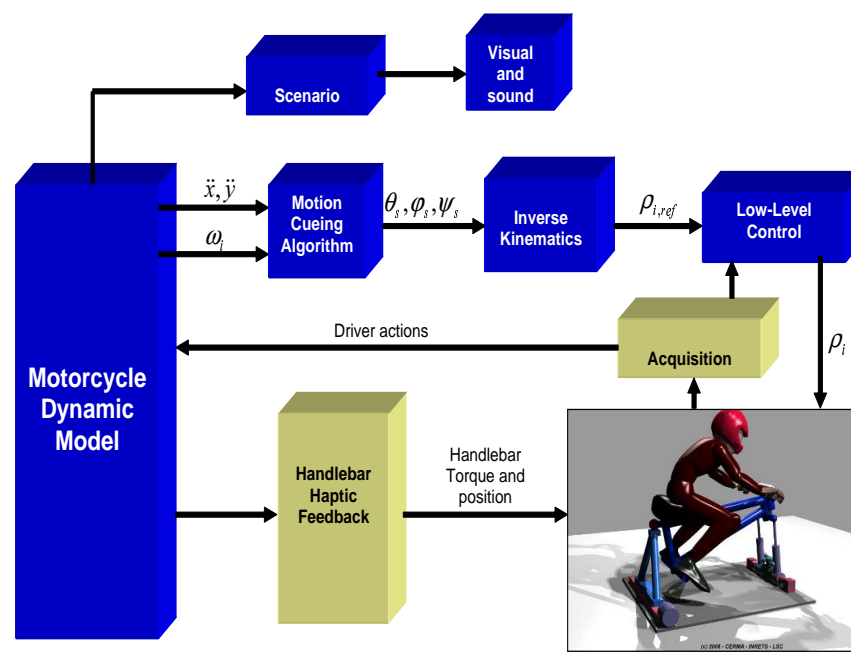

Fig. 4. Simulator software architecture

2) Traffic, visual and acoustic module: It is one of the most important part of the simulation. It was the object of ARCHISIM project who aimed to implement a realistic simulation of road situations, starting from the individual driver behaviors [14]. ARCHISIM allows the simulation of a road traffic of several tens of moving objects in realtime. Thus, it is possible to immerse the driver in a realistic traffic and to install him under desired conditions. The visual system is based mainly on SGI Performer library. The visual cue is realized by three BARCO projectors and three adjacent screens giving a large visual field. The restituted sounds are mainly those of the controlled virtual vehicle (engine mode) and of the traffic environment.

\section{Motion planning and control PC}

The simulator platform should be able to reproduce the motorcycle dynamics as faithfully possible as that caluclated by the dynamic model. However, because of the physical limits, the mechanical platform cannot reproduce the totality of the inertial forces (accelerations), present in real riding situations. Thus, a cueing algorithm is necessary to plan the platform trajectories which remain inside the atteignable workspace and that reproduce well riding behavior.

Three principal motion cueing strategies were developed and detailed in the literature that is classic Washout [15]
[16], the one based on optimal algorithm [17] and adaptive algorithm [18]. Recently a control strategy based on washout filters was proposed for a motorcycle riding simulator, whose kinematic structure is a 6 DOF Stewart platform [19].

As our simulator has 3 rotation DOF, we can not reproduce the transitory linear acceleration by translating the mobile platform. Based on our works on the driving simulator SIM ${ }^{2}$ [20], [21], we have chosen the classial control scheme by retrieving the translation canal and use only the tilt rendering as presented in the figure 5 .

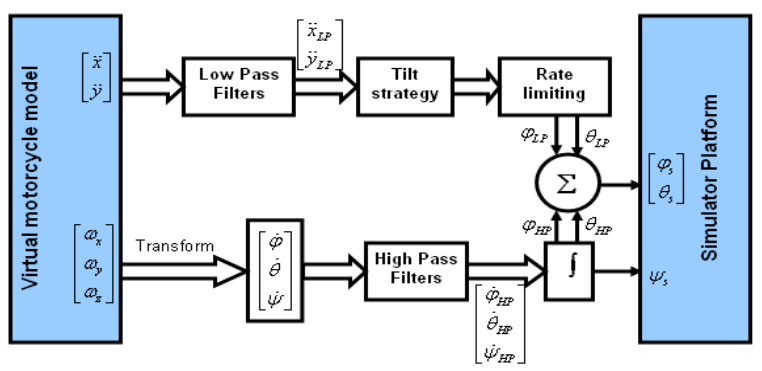

Fig. 5. Motion Cueing strategy

The inputs to the motion cueing strategy are the longitudinal, lateral accelerations and the three components of the rotation velocity as calculated by the virtual motorcycle model. The linear accelerations are low pass filtered to extract the maintained componants and the resulting signals are exploited to determine repectively pitch and roll tilt angles as follow:

$$
\begin{aligned}
& \theta_{t i l t}=\arcsin \left(\frac{\ddot{x}_{L P}}{g \cos \varphi}\right) \\
& \varphi_{t i l t}=-\arcsin \left(\frac{\ddot{y}_{L P}}{g}\right)
\end{aligned}
$$

where $\ddot{x}_{L P}, \ddot{y}_{L P}$ are the low pass component of $\ddot{x}, \ddot{y}$ and $g$ is the gravity vector.

The rotation velocity of the virtual motorcycle is firstly transformed into a Euler angles rates, high pass filtered and integrated. The resulting signals are added to the tilt angles to give the simulator orientation angles. However, since the tilt is just an artifact to trick the driver, the corresponding rates are to be limited (generally at $3^{\circ} / \mathrm{s}$ ) to be not detected bythe driver's vestibule.

\section{Performance Validation tests}

\section{A. platform actuation system}

Some tests were carried out in open-loop (without driver actions) to validate the actuation system performances (figure 6). In this test we aim to demonstrate the capabilities of the simulator platform to reproduce accelerations and angular velocities of two basic maneuvres: an acceleration/braking and constante velocity turn maneuvre (figure 7). Experimentations are realized in open-loop mode without rider on the simulator. 


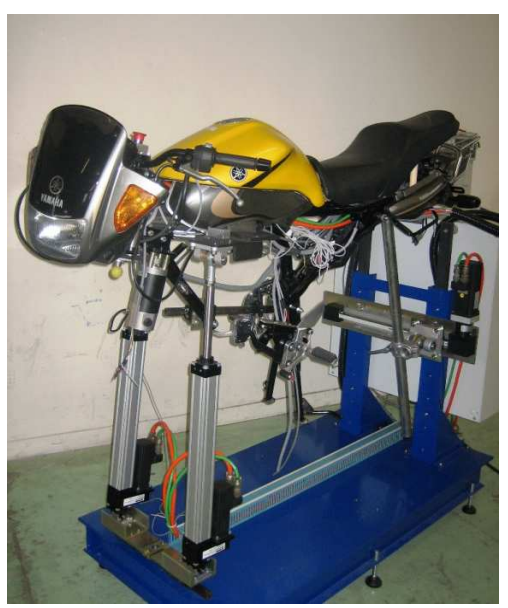

Fig. 6. Constructed riding motorcycle simulator

A $10 s$ motorcycle turn trajectory with constante forward velocity is created which consists of a three parts: $2.5 \mathrm{~s}$ of entering curve, $5 \mathrm{~s}$ of a $0.5 \mathrm{rad}$ roll angle and $2.5 \mathrm{~s}$ for exit. The second maneuvre is an acceleration start-up of $5 \mathrm{~m} / \mathrm{s}^{2}$ during $2 s$ and a braking deceleration of $-5 \mathrm{~m} / \mathrm{s}^{2}$ during $2 s$. This two maneuvres are fed back to the dynamic motorcycle model to determine all the motorcycle quantities (lateral acceleration, angular accelerations and velocities).

A Proportional-Derivative (PD) controller is used to stabilize the motorcycle by calculating the input handlebar's torque $T_{r}$ exerted by the rider from the desired roll angle $\varphi_{d}$ and the current roll angle $\varphi$ and rate $\dot{\varphi}$ as: $T_{r}=k_{p}\left(\varphi_{d}-\right.$ $\varphi)+k_{d} \dot{\varphi}$, where, $k_{p}, k_{d}$ are proportional and derivative gains. Finally, with the motion cueing algorithm, we deduce the desired reference angles to actuate the simulator's platform.

Figure 8 presents the lateral and longitudinal acceleration reproduced at the rider's head reference by the motorcycle simulator as a response to the real motorcycle turn and acceleration/braking maneuvres respectively. We show that the simulator is able to reproduce the low frequency components of the motorcycle acceleration by tilting the platform. It has to be noted that the maximum allowed platform rotations are for $\pm 15^{\circ}$ which means that in the best cases we can't restitute a maintaned linear acceleration over to $2.54 \mathrm{~m} / \mathrm{s}^{2}$.
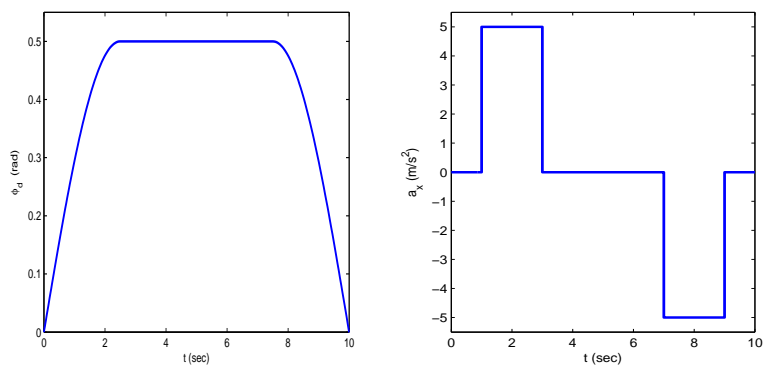

Fig. 7. (a) $\varphi_{d}$ of the turn and (b) acceleration/braking maneuvres
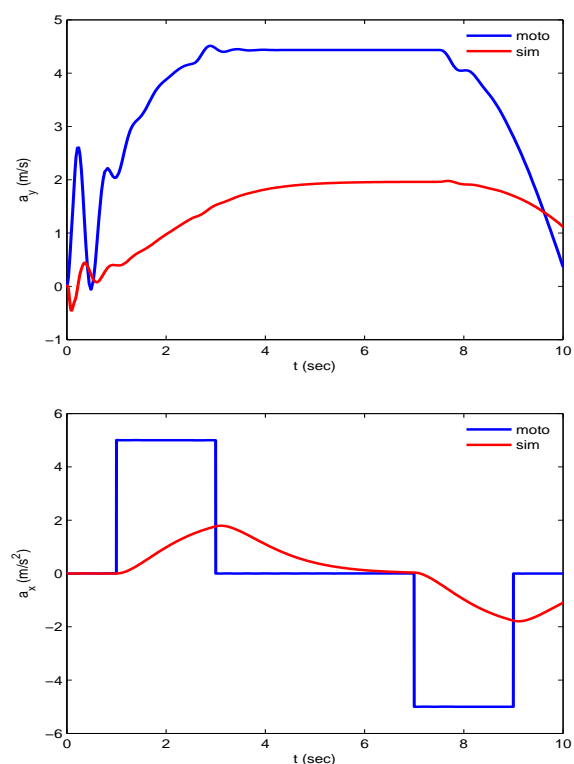

Fig. 8. Real and simulator sensed head: (a) lateral acceleration for turn maneuvre, (b) longitudinal acceleration for acceleration/braking maneuvre

However, for the accelerations high frequency components, we must translate the upper simulator's platform linearly in the desired direction. As we previously explained, the present platform has 3 rotations DOF without independent translations. Unfortunately, we must just emulate the high frequency canal by platform rotations.
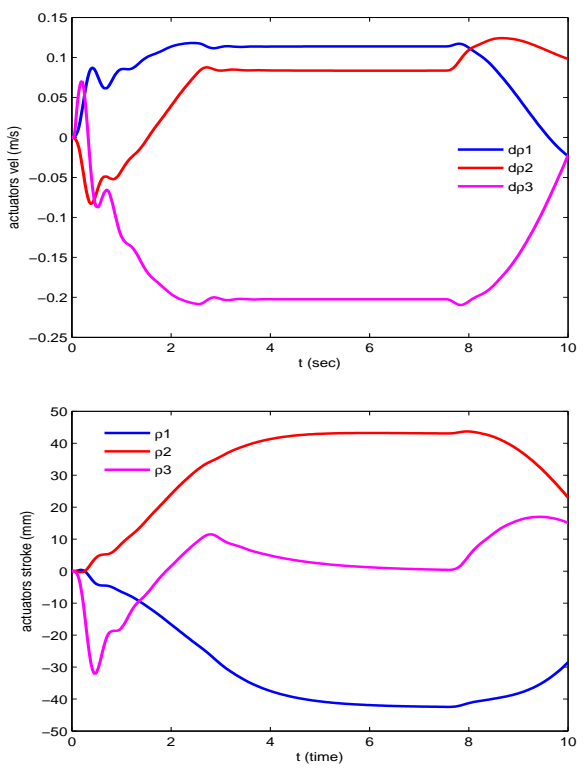

Fig. 9. Measured actuators velocities and strokes for turn maneuvre

Measured actuators velocities and strokes are shown in figure 9 for the turn maneuvre and figure 10 for the startup and braking maneuvre. As mentionned in the previous section of system performance validation, the different actuators quantities (stroke and velocity) are in the allowable workspace. 

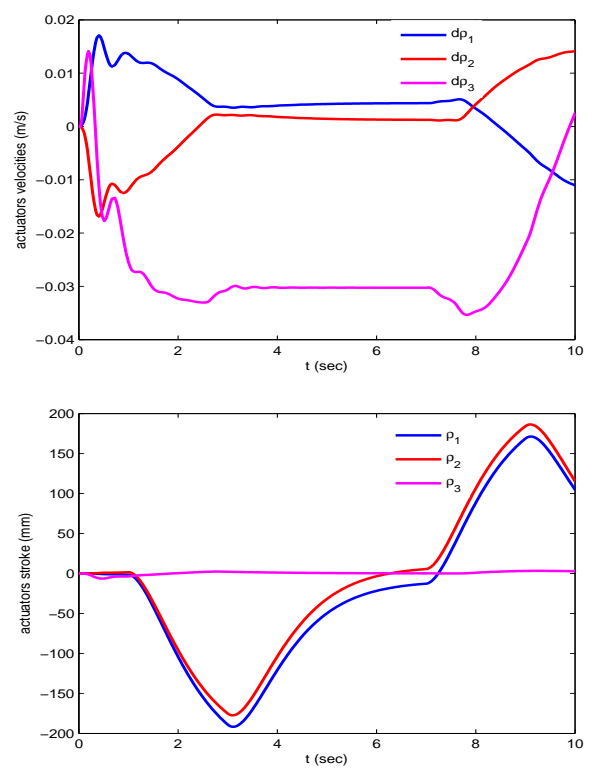

Fig. 10. Measured actuator velocities and strokes for acceleration/braking maneuvre

\section{CONCLUSION AND FUTURE WORKS}

In the first part of this paper, the important points retained to conceive a two wheels riding simulator have been listed. The application to which it is dedicated and the necessary perception guided us to choose the mechanical architecture.

The inverse kinematics of the platform was presented allowing to transform the motion cueing algorithm trajectories into actuator inputs. The simulator software architecture part including traffic, dynamic motorcycle model and motion cueing algorithm is illustrated.

The validation tests of performances are satisfactory and permits to reach our objectives for normal, dangerous or extreme situations. However, this judgment is taken in a control viewpoint. For a complete validation, psychophysical test for more different scenarios are of important necessity to answer many questions such that, the washout location, the produced movement fidelity and the realism of the riding simulation.

Future works are foreseen which will focus on tests of in the closed-loop simulation including the a rider on simulator. A more complex motorcycle model will be validated by real data record in real riding situations and will be integrated to the simulation loop. The double kinaesthetic feedback on the handlebar is about to be integrated to the suited motion restitution algorithms. Moreover, the present simulator will be embarked on the SIM $^{2}$ driving simulator platform $( \pm 60 \mathrm{~cm})$ to explore either longitudinal and lateral DOF on the linear acceleration restitution during the riding simulation.

\section{ACKNOWLEDGMENT}

This research was supported by funds from the NATIONAL RESEARCH AgENCY in Simacom project.

\section{REFERENCES}

[1] Les motocyclettes et la sécurité routiére en France en 2003. La documentation Francaise, Observatoire National Interministriel de Sécurité Routiére, ISBN: 2-11-005951-6, 2005.

[2] Y. Miyamaru, G. Yamasaki, and K. Aoki, "Development of a motorcycle riding simulator," Society of Automotive Engineers of Japan, vol. 23, pp. 121-126, 2002.

[3] T. Katayama, "A simulation model for riders control behavior," Society of Automotive Engineers of Japan, no. 961, 1996.

[4] S. Chiyoda, K. Yoshimoto, D. Kawasaki, Y. Murakami, and T. Sugimoto, "Development of a motorcycle simulator using parallel manipulator and head mounted display," in Driving Simulation Conference(DSC00), Paris,France, 2000.

[5] D. Ferrazzin, F. Barnagli, C. Avizzano, G. Pietro, and M. Bergamasco, "Designing new commercial motorcycles through a highly reconfigurable virtual reality-based simulator," Journal of Advanced Robotics, vol. 17, no. 4, pp. 293-318, 2003.

[6] V. Cossalter, A. Doria, and R. Lot, "Development and validation of a motorcycle riding simulator," in World Automotive Congress(FISITA2004), Barcelona, Spain, May 2004.

[7] J. Neimer, H. Mohellebi, S. Espié, and A. Kheddar, "Optimization of linear motion base dedicated to normal driving conditions," in Driving Simulator Conference (DSC05), Orlando, Florida, 30 nov-2 dec 2005.

[8] H. Mohellebi, S. Espié, and A. Kheddar, "Adaptive haptic steering wheel for driving simulators," in Proceedings of 2004 IEEE/RSJ International Conference on Robots and Intelligent Systems (IROSO4), June 2004.

[9] G. Yamasaki, K. Aoki, Y. Miyamaru, and K. Ohnuma, "Development of motorcycle training simulator," Society of Automotive Engineers of Japan, vol. 19, pp. 81-85, 1998.

[10] V. Cossalter, Motorcycle Dynamics. Milwaukee, USA: Race Dynamics Inc, ISBN: 0-9720514-0-6, 2002.

[11] H. Mohellebi, S. Espié, H. Arioui, A. Amouri, and A. Kheddar, "Low cost motion platform for driving simulator," in 5th International Conference on Machine Automation (ICMA04), Osaka, Japan, Nov. 2004.

[12] L. Nehaoua, A. Amouri, and H.Arioui, "Classic and Adaptive Washout Comparaison for a Low Cost Driving Simulator," in Proceedings of the 13th Mediterranean Conference on Control and Automation (MED05), Limassol, Cyprus, June 2005, pp. 586-591.

[13] R. Sharp, "Vibrational modes of motorcycles and their design parameter sensitivities," in Vehicle NVH and Refinement, Mechanical Engineering Publications, London, England, 1994, pp. 107-121.

[14] S. Espie, "Vehicle-driven simulator versus traffic-driven simulator: the inrets approach-driving simulation conference," in Driving Simulation Conference (DSC99), Paris, France, 1999.

[15] S. F. Schmidt and B. Conrad, "Motion drive signals for piloted flight simulators," in Contractor Report NASA CR-1601, Washington, USA, 1970.

[16] P. R. Grant and L. D. Reid, "Motion washout filter tuning: Rules and requirements," Journal Of Aircraft, vol. 34, pp. 145-151, Mar.-Apr. 1997.

[17] R. Telban and F. Cardullo, "A nonlinear human centred approach to motion cueing with neurocomputing solver," in AIAA Modeling and Simulation Technologies Conference and exhibit, Monterey, California, Aug. 2002.

[18] R. V. Parrish, J. E. Dieudonne, R. L. Bowles, and D. J. Martin, "Coordinated adaptive washout for motion simulators," Journal of Aircraft, vol. 12, pp. 44-50, July 1975.

[19] F. Barbagli, D. Ferrazzin, C. Avizzano, and M. Bergamasco, "Washout filter design for a motorcycle simulator," in Proceedings of VR2001, Yokohama, Japan, 2001, pp. 225-232.

[20] L. Nehaoua, H. Arioui, H. Mohellebi, and S. Espié, "Restitution Movement for a Low Cost Driving Simulator," in Proceedings of the 2006 American Control Conference (ACC06), Minneapolis, Minnesota, June 2006, pp. 2599-2604.

[21] L. Nehaoua, H. Arioui, H. Mohellebi, and S. Espié, "Motion Cueing Algorithms for Small Driving Simulator," in Proceedings 2006 IEEE International Conference On Robotics and Automation (ICRA06), Orlando, Florida, May 2006, pp. 3189-3194. 